\title{
The SFT-1 and OXA-1 respiratory chain complex assembly factors influence lifespan by distinct mechanisms in C. elegans
}

\author{
Sara Maxwell ${ }^{1}$, Joanne Harding ${ }^{2}$, Charles Brabin ${ }^{1}$, Peter J Appleford ${ }^{1}$, Ruth Brown ${ }^{1}$, Carol Delaney ${ }^{3}$, \\ Garry Brown ${ }^{1}$ and Alison Woollard ${ }^{1 *}$
}

\begin{abstract}
Background: C. elegans mitochondrial (Mit) mutants have disrupted mitochondrial electron transport chain function, yet, surprisingly, they are often long-lived, a property that has offered unique insights into the molecular mechanisms of aging. In this study, we examine the phenotypic consequences of reducing the expression of the respiratory chain complex assembly factors sft-1 (homologous to human SURF1) and oxa-1 (homologous to human OXA1) by RNA interference (RNAi). Mutations in human SURF1 are associated with Leigh syndrome, a neurodegenerative condition of the brain caused by cytochrome oxidase (COX) deficiency. Both SURF1 and OXA1 are integral proteins of the inner mitochondrial membrane, functioning in the COX assembly pathway.

Results: RNAi of both of these genes in C. elegans is associated with increased longevity, but the mechanism by which lifespan is extended is different in each case. sft-1 (RNAi) animals display lifespan extension that is dependent on the daf-16 insulin-like signaling pathway, and associated with sensitivity to oxidative stress. oxa-1(RNAi) animals, in contrast, exhibit increased longevity that is at least partially independent of daf-16, and associated with a reduced developmental rate and increased resistance to oxidative stress.

Conclusions: This study further delineates the consequences of mitochondrial dysfunction within a whole organism that will ultimately help provide new models for human mitochondrial-associated diseases. The difference in phenotype observed upon down-regulation of these two COX assembly factors, as well as phenotypic differences between these factors and other respiratory chain components analyzed thus far, illustrates the complex inter-relationships that exist among energy metabolism, reproduction and aging even in this simplest of metazoan model organisms.
\end{abstract}

Keywords: Mitochondria, Respiratory chain complex, Aging, Lifespan, C. elegans, SURF1, OXA-1

\section{Background}

Defects in mitochondrial function are implicated in a wide range of human diseases, affecting both development and the maintenance of normal structure and function [1]. The complexes of the mitochondrial respiratory chain are each composed of multiple subunits derived from both nuclear and mitochondrial genes. Because of the dual genetic contributions, it has proved difficult to generate model systems in which to study the

\footnotetext{
* Correspondence: alison.woollard@bioch.ox.ac.uk

${ }^{1}$ Biochemistry Department, University of Oxford, South Parks Road, Oxford OX1 3QU, UK

Full list of author information is available at the end of the article
}

consequences of specific mitochondrial defects, particularly those due to primary mutations in mitochondrial DNA (mtDNA). One way to approach this problem is to exploit the observations that a number of accessory proteins are required for the assembly of respiratory chain complexes in the inner mitochondrial membrane [2-4]. Deficiencies of these complexes, either singly or in combination, can be generated by targeted disruption of the nuclear genes for these assembly factors. In addition, defects in the assembly proteins often do not result in complete deficiency of the respiratory complexes and this is an advantage when studying key components of the main energy generating system of the cell, where

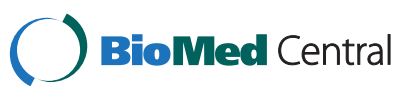

(c) Maxwell et al.; licensee BioMed Central Ltd. This is an Open Access article distributed under the terms of the Creative Commons Attribution License (http://creativecommons.org/licenses/by/2.0), which permits unrestricted use, distribution, and reproduction in any medium, provided the original work is properly cited. 
complete deficiency could result in early embryonic lethality.

The nematode worm, C. elegans is an established model organism for the study of pathogenesis of human mitochondrial diseases, exploiting the remarkable degree of evolutionary homology between both nuclear-encoded and mtDNA-encoded components of complexes of the respiratory chain, as well as associated assembly factors (reviewed in $[5,6]$. There are a number of reports of the consequences of defects in mitochondrial function in $C$. elegans (reviewed in $[7,8]$. These studies were often undertaken in the first instance to evaluate $C$. elegans as a potential model system for the investigation of pathogenic mechanisms in human mitochondrial disease; however, some unexpected phenotypic consequences of mitochondrial enzyme deficiencies were soon identified, particularly in relation to lifespan. While these are not immediately relevant to the human diseases, they may provide unique insights into the molecular mechanisms of aging $[8,9]$.

One of the most striking findings is that different mitochondrial defects, which result in comparable levels of impairment of energy generation, can have opposite effects on lifespan. For example, mutation in the isp-1 gene, encoding a Reiske iron sulphur protein that is a component of mitochondrial complex III, displays decreased mitochondrial respiration and significantly prolonged lifespan [10]. Likewise, $c l k-1$ mutants, lacking an enzyme required for ubiquinone synthesis (essential for complex I-dependent respiration), lrs-2 mutants (lacking a mitochondrial tRNA synthetase), and nuo-1 and atp-2 mutants, containing defects in mitochondrial proteins affecting complexes I and V, respectively, all display an increased lifespan [11-13]. Furthermore, various genome-wide RNAi screens have identified a wide range of mitochondrial genes that, when silenced, prolong lifespan $[10,12,14,15]$.

In contrast, the complex I subunit mutant gas-1 (fc210), has a shortened lifespan [16], as has the complex II subunit mutant mev-1(kn1) and the complex III subunit mutant ucr-2.3(pk732) $[9,17]$. Thus, there is no simple relationship between the respiratory chain complex targeted and the phenotypic outcome. An early assumption in the field, based on the "free radical theory of ageing" was that oxidative stress would play a key role in the altered longevity of mitochondrial mutants, with impaired mitochondrial function lowering the level of reactive oxygen species (ROS) and, hence, increasing lifespan. Alternatively, interfering with the function of respiratory chain complexes can in some situations lead to increased ROS and, therefore, shorten lifespan. However, extensive investigations have collectively revealed that oxidative damage can be experimentally dissociated from aging (reviewed in [8]). For example, slow physiological rates and prolonged lifespan were not found to be associated with increased stress resistance or reduced oxidative damage in Clk mutants that displayed mitochondrial dysfunction and evidence of decreased energy utilization [18]. Furthermore, oxidative stress was shown to actually increase worm lifespan in particular experimental conditions [19-22] and it has been proposed that increased ROS levels can lengthen lifespan via increased HIF-1 activity (reviewed in [23]), or by signaling to other endogenous defense mechanisms as part of an adaptive response (reviewed in [24]. Thus, it will be necessary to compare the functions of many different mitochondrial components, identifying common and conflicting features, to determine key elements in the relationship between mitochondria and aging.

In this study, we compare the consequences of the deficiency of two mitochondrial respiratory chain assembly factors, the protein products of the $s f t-1$ and oxa-1 genes. Targeted silencing of these $C$. elegans genes by RNA interference (RNAi) produces model deficiencies of these proteins in a tractable system, where the phenotypic consequences can be easily defined. The gene $s f t-1$ is the C. elegans ortholog of the human SURF1 gene. Mutations in SURF1 result in a systemic cytochrome oxidase deficiency; however, the mutant phenotype is restricted to the brain. Patients typically present with Leigh syndrome, a subacute neurodegenerative condition with characteristic necrotic lesions of the basal ganglia and brain stem $[3,25]$. The SURF1 gene product is an integral protein of the inner mitochondrial membrane, part of a $250 \mathrm{kDa}$ complex which appears to act early in the COX assembly pathway [26]. Patients with SURF1p deficiency do have some residual COX activity (5 to $10 \%$ ) indicating that some complex assembly can occur in the absence of this protein.

The human OXA1 and C. elegans oxa-1 genes encode the protein OXA1, a ubiquitous component of the inner mitochondrial membrane that inserts mitochondrially synthesized cytochrome oxidase subunits COX1, COX2 and COX3 into the inner membrane co-translationally, via binding of its $\mathrm{C}$-terminal domain to the mitochondrial ribosome [27]. Human OXA1 deficiency may be embryonic lethal, as no patients have yet been described.

Although both SURF1/SFT-1 and OXA-1 are components of the inner mitochondrial membrane that function in the assembly of respiratory chain complexes, the consequences of $s f t-1$ and oxa-1 knockdown in $C$. elegans are quite different. The overall result of knockdown of either gene is increased lifespan, but the mechanism by which prolonged lifespan is conferred, in terms of resistance to oxidative stress and dependence on the DAF-16/FoxO insulin-like signaling pathway component, are different, suggesting that distinct molecular pathways are involved. 


\section{Results}

\section{Decreased brood size in sft-1(RNAi) and oxa-1(RNAi)} worms

sft-1 (gene H06IO4.2) and oxa-1 (gene C01A2.3) were identified as the closest $C$. elegans homologues of human SURF1 and OXA1L, respectively. sft-1 and SURF1 both contain a Surfeit Locus 1 domain of approximately 300 amino acids, displaying $34.1 \%$ identity (53.8\% similarity). In C. elegans, $s f t-1$ is represented by the single deletion allele ok2277. Human and C. elegans oxa1 genes both contain a $60 \mathrm{kDa}$ inner membrane protein domain of approximately 200 amino acids, displaying $21 \%$ identity (43\% similarity). RNAi of $s f t-1$ or oxa-1 leads to significantly reduced gene expression and consequent reduction in cytochrome oxidase activity as assayed in whole animals in the case of $s f t-1$ RNAi (Figure 1A,B). Knockdown of either gene was associated with decreased brood size (a 27\% decrease, on average, following sft-1 RNAi by injection, and a $57 \%$ decrease, on average, in worms injected with oxa-1 dsRNA, Figure 1C). Although similar phenotypes were observed when RNAi by feeding was performed, the penetrance of defects in the latter case was slightly lower (a 15\% reduction in the case of $s f t-1$ feeding RNAi and a $43 \%$ reduction in the case of oxa-1 feeding RNAi). In subsequent experiments, RNAi by injection was utilized as the more reliable method of choice.

$s f t-1$ (RNAi) animals developed at the same rate as WT N2 controls and did not exhibit embryonic lethality to any significant level, suggesting that the reduction in brood size is a consequence of reduced germ cell production. A similar reduction in brood size was noted for the F1 progeny of worms injected with $s f t-1$ dsRNA as for the injected mothers themselves. F2 progeny of injected mothers, as expected, had normal brood sizes (data not shown). The brood size of $s f t-1$ (RNAi) animals could be increased if $\mathrm{F} 1$ progeny from $s f t-1$ RNAi injected worms (at the L4 stage) were mated with WT males (data not shown), suggesting that defects in spermatogenesis are partly responsible for the lower brood size. However, supplying WT sperm did not restore brood sizes to normal, indicating that knockdown of $s f t-1$ leads to defects in both sperm and oocyte production.

oxa-1 RNAi resulted in a high proportion of embryonic lethality (around 17\% of the progeny of oxa-1 dsRNA injected animals, Figure 1C). Dead eggs showed a range of defects at various stages in embryonic development, including regions of necrotic death in gastrulating early embryos, posterior abnormalities and failure to elongate further than the 1.5-fold 'comma' stage (Figure 1D). Viable offspring of injected mothers displayed a very pronounced slow growth (Gro) phenotype compared to $\mathrm{N} 2$ controls, taking over twice as long to reach adulthood, (for N2 controls, $100 \%$ of hatchlings reached L4 (by the criteria of a visible vulva) after 48 hours. For oxa-1(RNAi) animals, 0\% of hatchlings reached L4 by 48 or 72 hours, and typically only $50 \%$ of hatchlings reached L4 (or at least some aspects of L4) after 96 hours). F1 progeny of oxa-1(RNAi) animals produced almost no progeny of their own.

\section{Increased lifespan in sft-1(RNAi) and oxa-1(RNAi) worms}

Cumulative survival curves of the F1 offspring of injected sft-1(RNAi) worms show an extended lifespan compared with the WT control (Figure 2A). Mean lifespan from $\mathrm{L} 4$ was $17.7 \pm 0.6$ days for $s f t-1$ (RNAi) worms $(\mathrm{n}=95)$ and $15.1 \pm 0.5$ days for $\mathrm{N} 2$ control $(\mathrm{n}=79)$, with $50 \%$ survival at days 19 and 15 , respectively and maximal lifespans of 27 days for $s f t-1$ (RNAi) animals and 23 days for WT (the whole data set is shown in Additional file 1). With the F1 offspring of injected oxa1(RNA $i$ ) worms, the lifespan was extended even further with a mean of $19.3 \pm 0.8$ days $(\mathrm{n}=88)$, a maximum of 31 days and 50\% survival at Day 20 (Figure 2A, Additional file 1). Experiments were begun with animals at the L4 stage, although it was difficult to precisely stage oxa-1(RNAi) animals given the developmental delay. It is possible that further developmental delay (that is, in progressing from L4 to adulthood) could contribute to the lifespan extension, although given that oxa-1(RNAi) animals, on average, took twice as long to reach adulthood, the delay from L4 to adulthood would only be expected to contribute to lifespan extension by a maximum of one day, and observed lifespan extension was much more pronounced than this.

To ascertain whether the lifespan extension was dependent on the daf-16 insulin-like signaling pathway, RNAi of $s f t-1$ and $o x a-1$ by injection was performed in a daf-16(m26) mutant background, which has previously been shown to suppress the lifespan extension of both daf-2(e1370) and age-1(hx546) mutants [28,29]. In a daf16(m26) mutant background, sft-1 RNAi resulted in mean and maximum lifespans of $13.5 \pm 0.4$ days $(\mathrm{n}=92)$ and 21 days respectively, very similar to daf-16 controls (13.6 \pm 0.4 days $(\mathrm{n}=92)$, maximum 19 days) (Figure 2B, Additional file 1). This suggests that lifespan extension in $s f t-1$ (RNAi) worms is dependent upon the daf-16 pathway, and that $s f t-1$ may operate upstream of daf-16 to regulate longevity.

In the case of oxa-1 RNAi in a daf-16 mutant background, however, a different result was obtained. In this case, lifespan was still extended following oxa-1 knockdown, with a mean lifespan of $17.9 \pm 0.7$ days $(n=84)$ and maximum lifespan of 31 days, not statistically different to the data obtained when oxa-1 RNAi is performed in a wild type background (Figure 2C, Additional file 1). This suggests that lifespan extension caused by oxa-1 


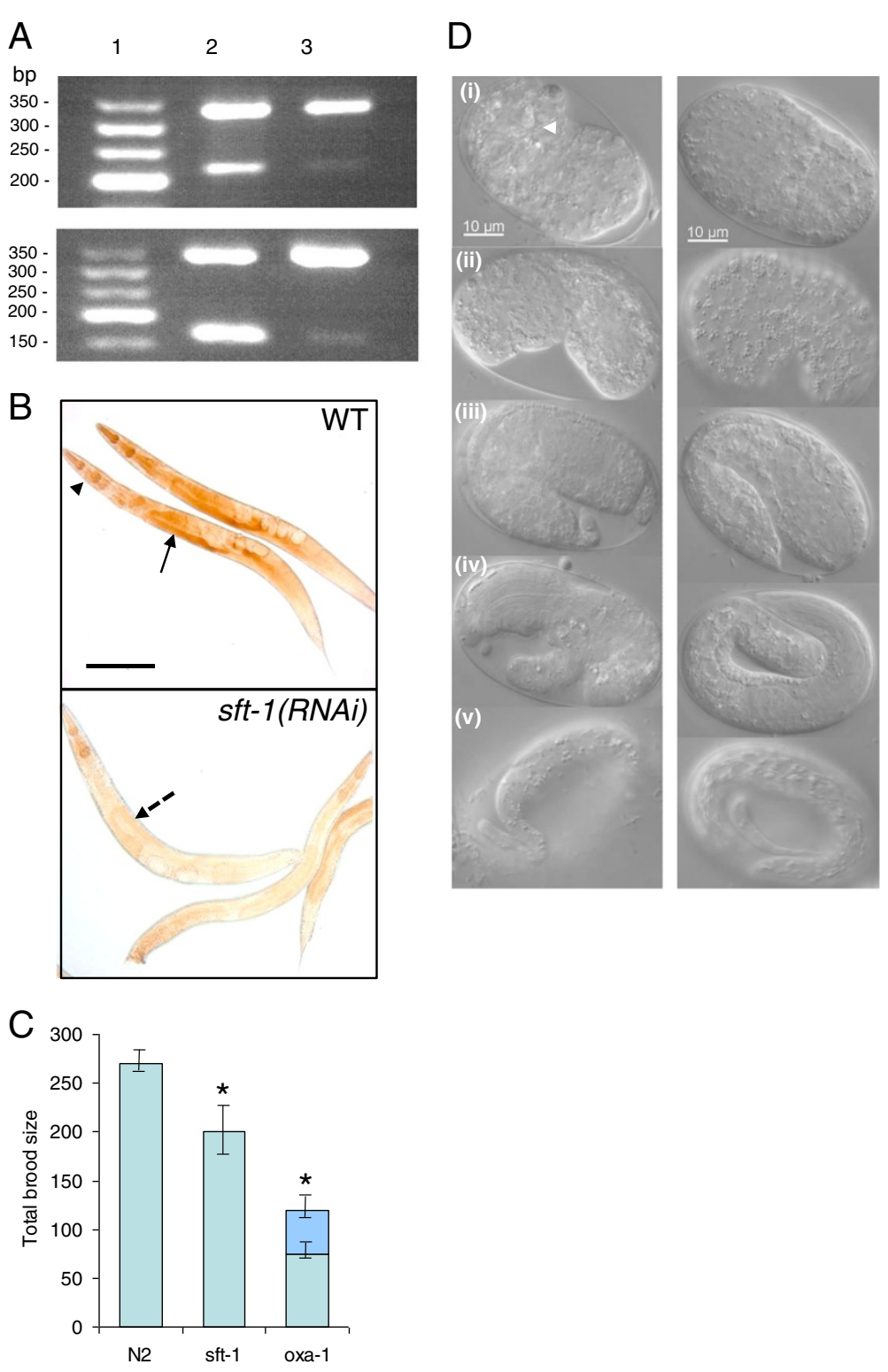

Figure 1 sft-1 or oxa-1 RNAi results in reduced gene expression, COX staining and brood size. A: RT-PCR of oxa-1 and sft-1 to compare mRNA levels. Lane 1, 50 bp DNA ladder. Upper panel: Lane 2, RT-PCR products from WT animals. Upper band (350 bp) corresponds to eft-2 mRNA (internal control), lower band (220 bp) corresponds to sft-1 mRNA. Lane 3, RT-PCR products from the progeny of animals injected with sft-1 dsRNA. sft-1 expression is greatly reduced. Lower panel: Lane 2, RT-PCR products from WT animals. Upper band (350 bp) corresponds to the eft-2 internal control, lower band (164 bp) corresponds to oxa-1 mRNA. Lane 3, RT-PCR products from the progeny of animals injected with oxa-1 dsRNA. oxa-1 expression is greatly reduced. B: COX staining in WT and sft-1(RNAi) animals. In WT, COX staining is particularly prevalent in the pharynx (black arrowhead) and germ line (black arrow), tissues that have high energy demands. In sft-1(RNAi) animals, COX staining is greatly reduced (photomicrographs taken using identical exposure times), especially in the germ line (dotted arrow). Scale bar, $100 \mu \mathrm{m}$. C: Brood size of WT animals injected with sft-1 or oxa-1 dsRNA compared with control animals injected with TE only (labeled N2). Both sft-1 and oxa-1 RNAi result in a significantly reduced brood size compared with controls $\left({ }^{*} P<0.005\right)$. The darker shaded area of the bar corresponding to oxa-1(RNAi) represents the proportion of dead embryos. Error bars denote the standard error of the mean (SEM). D: Embryonic lethality induced by oxa-1 RNAi. Left hand panel: oxa-1 (RNAi) embryos, right hand panel: WT embryos at equivalent stages. Defects can be seen throughout embryogenesis, including necrotic regions in early embryos (i, white arrowhead), morphological defects at the "bean" stage (ii), elongation defects at the "comma" stage (iii) and later (iv, v). Posterior defects are particularly pronounced. 

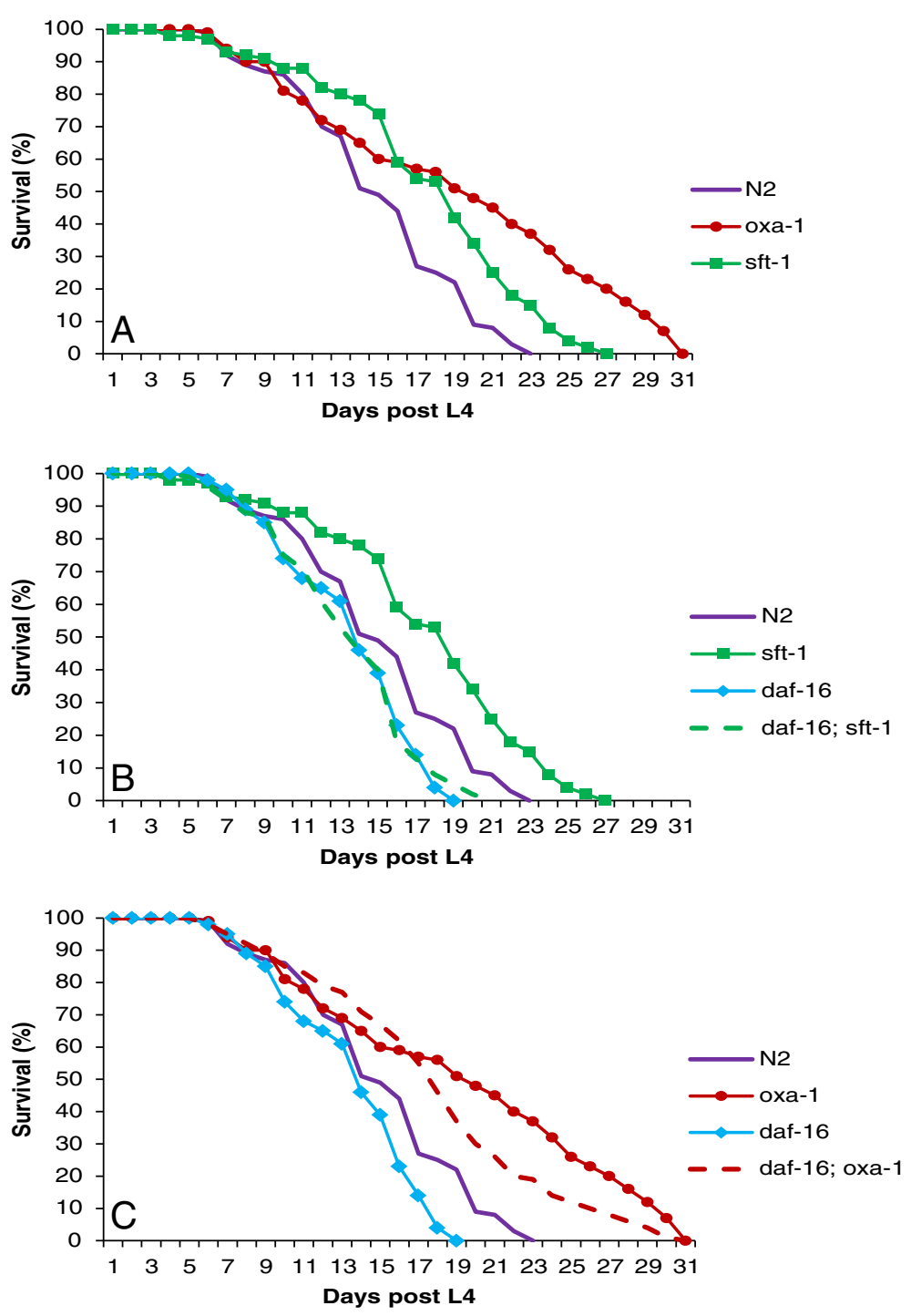

Figure 2 Lifespan extension in sft-1(RNAi) and oxa-1(RNAi) animals. A: Lifespan curves for N2, oxa-1(RNAi) and sft-1(RNAi) animals (recorded as number of days of survival post $L 4$, or the time at which controls reached $L 4$, in the case of oxa-1(RNAi) animals). Lifespan is prolonged in sft-1 (RNAi) and oxa-1 (RNAi) animals compared with WT controls, with oxa-1 having the greatest effect. B: Lifespan analysis of sft-1(RNAi) animals performed in a daf-16(m26) mutant background, in which sft-1 RNAi fails to extend lifespan. C: Lifespan analysis of oxa-1 (RNAi) animals performed in a daf-16(m26) mutant background. oxa-1 RNAi still extends lifespan under these circumstances. The data presented combine three independent data sets (the data for individual replicate experiments can be found in Additional file 1).

knockdown is independent of the daf-16 insulin-like signaling pathway. However, we do note that the shape of the lifespan curves is somewhat different when oxa-1 (RNAi) animals and daf-16; oxa-1(RNAi) animals are compared, and that the $50 \%$ survival point is reduced from 20 to 17 days, respectively. This may suggest a somewhat complex interaction between oxa-1 and daf16 , or that suppression is incomplete with the daf-16 allele used in this analysis, and caution needs to be exercised in the interpretation of this result. What is clear, however, is that the two knock-downs produce different phenotypes in terms of embryonic lethality, brood size and developmental delay, and respond somewhat differently to perturbations in the daf-16 pathway, with $s f t-1$ showing complete dependence on functioning daf16 for lifespan extension, and the oxa-1(RNAi) phenotype being at least partially daf-16-independent.

It has previously been reported that, in the case of genes involved in mitochondrial function, the dose of dsRNA delivered to C. elegans in RNAi feeding experiments may affect the phenotypic outcome, with lower doses of particular dsRNAs causing lifespan extension while higher doses shorten lifespan [9]. It is well established that RNAi by injection tends to give more 
severe phenotypes than RNAi by feeding. In our experiments, we used undiluted dsRNA for injections, presumably representing the highest possible dose of RNAi. In addition, feeding RNAi experiments also resulted in lifespan extension (SM and AW, unpublished observations), suggesting that in the case of $s f t-1$ and $o x a-1$, the phenotypic outcome is unlikely to be dose-dependent (at least in terms of different doses resulting in opposite phenotypes).

\section{Response to oxidative stress following sft-1 and oxa-1 knockdown}

To investigate whether the lifespan extension was associated with resistance to oxidative stress, $s f t-1(R N A i)$ and oxa-1(RNAi) worms were subjected to oxidative stress treatment with the herbicide paraquat. This causes oxidative stress by a metabolically catalyzed reaction resulting in depletion of NADPH and production of ROS, primarily superoxide anions [30].

The resistance to oxidative stress of the $s f t-1$ (RNAi) worms was not significantly different from the N2

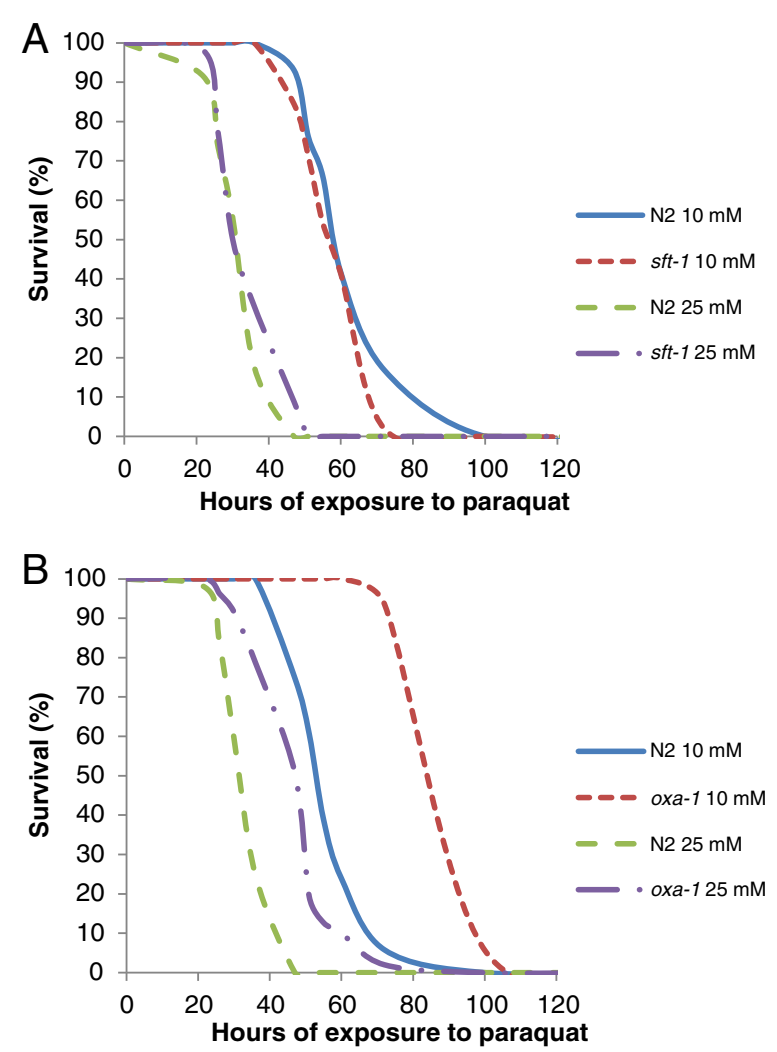

Figure 3 Sensitivity of sft-1(RNAi) and oxa-1(RNAi) animals to oxidative stress. A: Survival curves of WT or sft-1(RNAi) animals exposed to $10 \mathrm{mM}$ or $25 \mathrm{mM}$ paraquat, recorded as the number of hours of survival following exposure. WT and stt-1 (RNAi) animals display similar sensitivity to paraquat at either concentration. B: Survival curves of size-matched WT and oxa-1(RNAi) animals exposed to paraquat. oxa-1 (RNAi) animals show marked resistance to paraquat, at both $10 \mathrm{mM}$ and $25 \mathrm{mM}$ concentrations. controls at either 10 or $25 \mathrm{mM}$ paraquat (Figure $3 \mathrm{~A}$ ), with mean survival time following exposure of $s f t-1$ (RNAi) animals to $10 \mathrm{mM}$ paraquat of $61.3 \pm 2$ hours ( $\mathrm{n}=36$ ), compared to $65 \pm 3.1$ hours for WT controls ( $\mathrm{n}=29$ ). Similarly, exposure to $25 \mathrm{mM}$ paraquat resulted in a mean survival time of $35.4 \pm 1.5$ hours for $s f t-1$ (RNAi) animals $(\mathrm{n}=36)$ compared with $33.8 \pm 1.3$ hours for WT controls $(\mathrm{n}=35)$ (Additional file 2). By contrast, the oxa-1(RNAi) worms were much more resistant to paraquat than the control worms at both concentrations (Figure 3B). Given the Gro phenotype of oxa-1(RNAi) animals and, therefore, the time it takes for them to reach L4 (when our paraquat survival assays normally start), we used three-day-old progeny of oxa-1 dsRNA injected hermaphrodites, and size-matched WT controls (L1s), to control for the possibility that animal size could have an effect on paraquat sensitivity that could complicate our results. In this case, exposure to $10 \mathrm{mM}$ paraquat resulted in a mean survival time of $58.4 \pm 1.9$ hours for WT $(\mathrm{n}=38)$ and $100.1 \pm 1.9$ hours for oxa-1(RNAi) animals $(n=42)$. Increasing the concentration of paraquat to $25 \mathrm{mM}$ gave similar results, with a mean survival time of $35.9 \pm 1.2$ hours for WT $(\mathrm{n}=39)$ and $49.7 \pm 1.9$ hours for oxa-1(RNAi) animals $(\mathrm{n}=48)$ (whole data set shown in Additional file 3). In the case of the oxa-1 (RNAi) animals used for this experiment, it is difficult to categorically state what developmental stage they were at. They were size-matched to L1 control WT animals, but may have been more advanced in terms of certain aspects of development, which could be uncoupled when $o x a-1$ is knocked down and overall growth slows. In any case, however, it is noteworthy that WT animals did not appear to display any size-dependent resistance to paraquat (mean survival time of $65 \pm 3.1$ hours for L4 animals following exposure to $10 \mathrm{mM}$ paraquat, compared with $58.4 \pm 1.9$ for L1s, and $33.8 \pm 1.3$ hours for L4s compared with $35.9 \pm 1.2$ hours for L1s exposed to 25 $\mathrm{mM}$ paraquat), indicating that animal size differences are unlikely to be confounding our data.

\section{OXA-1 and SFT-1 tissue distributions oxa-1::GFP}

The expression pattern of a translational oxa-1::gfp fusion construct is shown in Figure 4. oxa-1::gfp is expressed at high levels throughout the animal. oxa-1:: $g f p$ expression is widely distributed, showing a punctate mitochondrial network pattern, and is particularly prominent in pharyngeal and body wall muscle (Figure 4). Mitochondria form elongated filaments or networks in many cell types that is particularly prominent in tissues with a high energy demand, such as muscle. To demonstrate that the expressed product did indeed localize to mitochondria, oxa-1::gfp worms were stained with Mitotracker Red and extensive co-localization was 


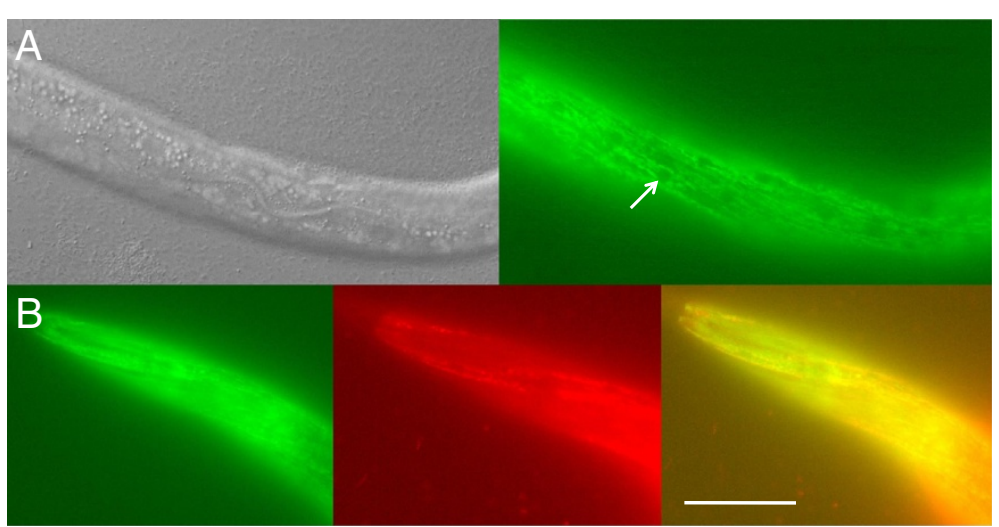

Figure 4 oxa-1::gfp expression pattern. A, B: Transgenic strain AW241 (ouEx609 (pAW318 + rol-6)), where pAW318 is a translational GFP fusion of oxa-1 driven by the endogenous promoter. oxa-1 is expressed at a high level throughout the worm in a reticular mitochondrial network pattern characteristic of mitochondrial proteins. A: expression of oxa-1::gfp is particularly strong in body wall muscle cells, where it is excluded from the nuclei (white arrow). B: co-localization of oxa-1::gfp (left hand green image) and Mitotracker Red (central red image) in the merged image (right hand yellow image), confirming expression of oxa-1::gfp in mitochondrial networks. Scale bar, $100 \mu \mathrm{m}$. Anterior is to the left in all images.

observed (Figure 4B). Embryonic oxa-1::gfp expression is very faint, but observable at the 100 cell stage (data not shown).

\section{sft-1::gfp}

The $s f t-1$ gene appears to be contained within a large operon, CEOP3088, making the construction of reporter constructs problematic, as it is difficult to predict where the important regulatory elements will be and the required constructs are likely to be very large (CEOP3088 covers some $40 \mathrm{~Kb}$ ). Recent analysis suggests that $s f t-1$ is part of a "hybrid" operon, however, containing intercistronic promoter/enhancer elements required for tissue-specific gene expression [31]. Given the relatively large intercistronic region in the case of $s f t-1(>1.5 \mathrm{~Kb})$, we made a translational reporter construct in which this region was used to drive $s f t-1:: g f p$, which would be expected to report on at least part of the $s f t-1$ expression pattern. The expression pattern obtained with this construct is shown in Additional file 4. $s f t-1:$ GFP is expressed at a very low level throughout the worm with higher levels of expression in a region of body wall muscle adjacent to the pharyngeal bulb (Additional file 4A) and the uterine area (Additional file 4B). The low overall level of expression may well reflect the absence of operon-upstream regulatory elements from the $s f t-1:: g f p$ construct.

\section{Discussion}

Cytochrome oxidase deficiency generated by RNAi of sft-1 resulted in lifespan extension accompanied by decreased germ cell production but no obvious slowdown of developmental rate, whereas the lifespan extension caused by RNAi of oxa-1 was associated with a pronounced slow growth phenotype and lethal defects in embryonic development. The combination of dead embryos and long-lived progeny in the broods of oxa-1 (RNAi) animals could reflect some mitochondrial "threshold effect" in which the threshold for mitochondrial function is surpassed in some embryos, leading to catastrophic mitochondrial damage and the inability to invoke compensatory pathways.

The embryonic lethality and slow growth of viable progeny observed with RNAi of oxa-1 correlates with phenotypic observations of oxa-1 homologues in other organisms. Inactivation of both $O X A 1$ genes in S. pombe is lethal [32], whereas a knockout mutant of S. cerevisiae is viable, but is unable to respire and can only grow on fermentable carbon sources [4]. The function of these various OXA1 proteins is conserved, as $N$. crassa, human and $S$. pombe homologues can rescue $S$. cerevisiae oxa1 null mutants [33]. In yeast, defects in Oxa1 ablate the function of the cytochrome oxidase complex and greatly reduce levels of Complex III and $\mathrm{F}_{0} \mathrm{~F}_{1}$ ATPase [34]. Overall, the data suggest that OXA1 is important for the biogenesis of several different respiratory chain complexes (reviewed in [35]) and this may account for the severity of the oxa-1 RNAi phenotype seen in $C$. elegans. Consistent with this, no OXA1 deficient patients have been identified and this condition may thus be embryonic lethal in humans.

SURF1 deficient patients, on the other hand, usually survive into early childhood with a progressive neurodegenerative disease. In contrast to the human SURF1 deficient phenotype, no abnormal neuromuscular function was observed in $s f t-1$ (RNAi) worms, despite detailed observations of movement and pharyngeal pumping rates (data not shown). A possible caveat to this conclusion, 
however, is that RNAi in C. elegans is known to be relatively ineffective in the nervous system, thus neuronal phenotypes may have been masked in our experiments. It is also possible that the lack of a neuromuscular phenotype in the $s f t-1$ deficient worms may simply reflect overall energy requirements and the level of energy generation in different tissues. Previous studies of metabolic activity in C. elegans, as monitored by oxygen consumption, indicate that only a small proportion of total energy expenditure is on movement and the greatest single energetic demand is for reproduction [36]. There is a significant increase in oxygen consumption at the L3 and L4 larval stages, the time of extensive proliferation of germ cells (reviewed in [37]. Reflecting this dependence of reproduction on energy generating capacity, mutations leading to deficiency of two other components of the mitochondrial oxidative phosphorylation system, Complex I (nuo-1 gene) and the $\mathrm{F}_{1} \mathrm{~F}_{0}$ ATP synthase (atp-2 gene) also show a marked abnormality in gonadal development [13].

Decreased fecundity of $s f t$-1(RNAi) worms was attributed to a failure of both oogenesis and spermatogenesis, as unfertilized oocytes were not observed at a significant level and WT sperm was only able to partially restore the brood size. Consistent with the hypothesis that decreased energy generating capacity in $s f t-1$ (RNAi) worms due to COX deficiency may account for the reproductive failure, we observed high levels of COX activity in the germ line of WT animals. $s f t-1:: g f p$ was not observed in the germline, but a likely explanation for this is the phenomenon of gene silencing that is often triggered by transgene expression in the germline [38].

Both sft-1(RNAi) and oxa-1(RNAi) worms can be described as having Mit phenotypes (that is, those associated with mitochondrial dysfunction). Knockdown of either gene resulted in a significantly extended lifespan (particularly in the case of $o x a-1$ ) in at least two independent experiments, while only the oxa-1(RNAi) worms were resistant to oxidative stress induced by treatment with paraquat. The relationship between mitochondrial function and lifespan in C. elegans is complex, although most observations support two contributory mechanisms, nutritional restriction and damage due to ROS. Many different observations link nutritional/energetic status and lifespan in a wide range of different species. Food deprivation, either environmental or genetically determined (for example, eat-2 gene mutations [39]) and mutations in genes involved directly (for example, nuo-1 [13]) or indirectly (for example, $c l k-1$ [40]) in mitochondrial energy metabolism have all been shown to prolong life in C. elegans. These observations are incorporated in the "rate of living model" for longevity in which increased life expectancy is attributed to a generalized slowing down of metabolic function.
In nearly all cases examined thus far, lifespan extension in Mit mutants has been shown to act independently of the insulin/IGF signaling pathway [5]. Therefore, the dependence of $s f t-1(R N A i)$ animals on daf-16 for lifespan extension, suggesting that $s f t-1$ may act upstream of daf-16 to regulate longevity, is noteworthy, putting $s f t-1$ knockdown in a different phenotypic category from most other Mit phenotypes. The FoxO-like forkhead/winged helix transcription factor DAF-16 is thought to be a master regulator of aging, integrating metabolic signals, stress signals as well as reproductive signals from the germline to modulate longevity [41]. Perhaps the dependence of lifespan extension in $s f t-1$ (RNAi) animals on daf-16 reflects the relative importance of reduced fecundity in promoting longevity when $s f t-1$ expression is reduced.

The second model for the determination of lifespan in C. elegans is based on the accumulation of damage due to ROS, although recent analyses suggest a rather complex relationship between oxidative stress and aging, and one that can be experimentally uncoupled. The oxidative damage model is not independent of the rate of living model as the major site for the formation of ROS is the mitochondrion and production is related to the level of metabolic activity. Some Mit mutants (for example, lrs-2 and isp-1), in common with IGF/insulin signaling pathway mutants daf-2 and age-1, show increased lifespan and resistance to oxidative stress [12], reviewed in [8]. oxa-1, as presented here, appears to fall into this category. By contrast, gas-1, nuo-1 and mev-1 mutations confer reduced lifespan associated with a hypersensitivity to free radicals $[16,42,43]$, reviewed in [8]. Resistance to oxidative stress is suggested to extend lifespan by decreasing damage caused by accumulation of free radicals in the cell.

On the other hand, mutants such as $c l k-1$ and sod-2 display a hypersensitivity to oxidative stress but are also longlived [18,22], suggesting that oxidative stress per se is not always a primary cause of aging. Indeed, it has been suggested that ROS generated inappropriately in some mitochondrial mutants might actually extend lifespan by signaling various adaptive responses $[12,44]$. This proposed preconditioning of mitochondria has been termed mitohormesis [45]. Intriguingly, though, there do not appear to be mutants that are highly resistant to oxidative stress yet display a shortened lifespan, supporting the idea that oxidative stress resistance (or adaptation to oxidative stress) is associated with a long life.

"Oxidative stress resistance" is rather an umbrella term, as different Mit mutants display different sensitivities to a spectrum of oxidative stresses. For example, in one study of 10 different mutant/RNAi strains, most of the long-lived worms with compromised mitochondria displayed marked resistance to hydrogen peroxide, yet 
were not resistant (or even displayed increased sensitivity) to paraquat [12]. The authors of this study proposed that as paraquat triggers the production of superoxide in a NADPH-dependent reaction, paraquat hypersensitivity of worms with mitochondrial dysfunction might be due to an increase in NADPH associated with a particular metabolic response to reduced electron transport. However, the data that we present here indicate that oxa-1 (RNAi) worms, in contrast to those analyzed in the Lee study, exhibit marked resistance to paraquat. This suggests that it is very difficult to generalize about the effects of mitochondrial dysfunction, even within the group of mutants/RNAi-treated worms that display both prolonged lifespan and heightened stress resistance. Furthermore, a recent study has found that pathways of lifespan extension can differ depending on whether RNAi or mutation for the same gene is used to reduce gene function [46]. Mutations in sft-1 or oxa-1 were not analyzed here, but these would form an interesting subject for future analysis.

It has been previously suggested that long-lived Mit mutants utilize a novel metabolism, and that longevity in these animals may be dependent on this altered metabolic state. For example, it has been proposed, albeit using a limited number of mutants, that long-lived Mit mutants up-regulate fermentative malate dismutation, where fumarate is terminally reduced at complex II to succinate, generating fewer radical species overall [17]. This is an anaerobic metabolic pathway thought to be unique to nematodes, and normally up-regulated during dauer formation. However, other studies argue that although metabolic restructuring does indeed occur in Mit mutants, the restructuring per se does not cause lifespan extension [47]. Recent data, however, challenge this view. For example, the importance of the alternative glyoxylate pathway in Mit mutant lifespan extension has been investigated by knocking down gei-7, which encodes the main glyoxylate shunt enzyme in C. elegans. gei-7 mutation was found to suppress the enhanced longevity of $c l k-1$ mutants [48] and has also been found to reduce the lifespan of $c y c-1$ Mit mutants [49].

Strikingly, mitochondrial respiratory complex dysfunction models being developed in other systems display many of the same features as C. elegans Mit mutants. For example, a partial deficiency of Mclk1, the mouse $c l k-1$ ortholog, increases average lifespan by 30\% [50]. Even more relevant to this study, increased lifespan following inactivation of Surf1 in mice has been recently demonstrated [51], and similarly, Surf1 knockdown in the central nervous system of Drosophila melanogaster has also been shown to induce longevity [52]. This suggests that the function of $s f t-1$ in regulating lifespan is likely to be widely conserved, and it will be very interesting to discover the extent to which precise mechanisms of lifespan extension are conserved between disparate species. For example, it would be interesting to examine whether lifespan extension in $s f t-1$ knockdown animals is dependent on gei-7 and thus a shift to the glyoxylate pathway. It is not clear, however, how such metabolic restructuring might proceed in other organisms where this alternative pathway is not thought to operate.

Whatever the precise mechanisms, it is clear that $s f t-1$ and oxa-1 influence longevity through distinct molecular pathways, despite the fact that both genes encode factors required for COX assembly.

\section{Conclusions}

We have clearly shown that knockdown of $s f t-1$ or oxa-1 by RNAi results in increased longevity; however, distinct molecular mechanisms appear to operate in each case, and analysis of these two genes illustrate the principle that it is not possible to draw simple conclusions about the relationships between mitochondrial energetics, longevity, stress resistance and insulin-like signaling. Further analysis of COX deficiency and generation of worms with defects in other mitochondrial enzyme complexes will be required to further delineate the organismal consequences of mitochondrial dysfunction that will help provide new models for human mitochondrial-associated diseases. For the present, analysis of the effects of reducing expression of $s f t-1$ and oxa-1, both of which encode proteins required for the assembly of respiratory complexes, illustrates the diversity of phenotypic outcomes that can result from mitochondrial dysfunction and the complex interrelationships that exist between energy metabolism, reproduction and aging even in this simplest of metazoan model organisms.

\section{Methods}

\section{C. elegans strains and maintenance}

Experiments were performed with the wild-type (WT) Bristol strain N2 and daf-16(m26) mutant (provided by the Caenorhabditis Genetics Center). C. elegans strains were maintained and handled on nematode growth media (NGM) agar with E. coli OP50 as their food source [52]. Microscopy was carried out using a Zeiss Axiophot microscope (Carl Zeiss Ltd, Bicester, Oxon, UK) fitted with DIC and fluorescence optics as appropriate. Worms were anesthetized on $2 \%$ agarose pads as previously described [53].

\section{Bioinformatic analysis}

Homologs were identified by BLAST analysis (http://blast. ncbi.nlm.nih.gov). C. elegans protein sequences were retrieved from Wormbase (www.wormbase.org) and human protein sequences from the Ensembl database 
(www.ensembl.org). Sequences were analyzed using Pairwise alignment in Bioedit (www.mbio.ncsu.edu/bioedit/ bioedit.html).

\section{RNAi by injection}

PCR primers specific for $s f t-1$ (C. elegans gene H06I04.2) and oxa-1 (C01A2.3) and including T7 or T3 RNA polymerase promoter sites were designed. These amplified fragments of $679 \mathrm{bp}$ and $515 \mathrm{bp}$ were from genomic DNA, respectively.

sft-1 F primer 5' ATTAACCCTCACTAAAGTTATT TTGAAGC 3' JH1;

$s f t-1 \mathrm{R}$ primer 5'AATACGACTCACTATAGGTGACG GGGAATTC 3' JH2

oxa-1 F primer 5'ATTAACCCTCACTAAAGACA TTCCCTGGTGGGTTACA 3' JH5.

oxa-1 R primer 5'AATACGACTCACTATAGACGCA TAATTGGTGGCATTT 3' JH6

dsRNA was synthesized directly from PCR products as previously described [54] and injected into young adult hermaphrodites as previously described [55]. Injected worms were transferred to fresh seeded plates four hours after injection and the progeny assayed for relevant phenotypes, except in the case of brood size measurements, which were performed on the injected mothers themselves as well as the subsequent F1 progeny.

\section{RNAi by feeding}

L3 N2 (or daf-16(m26)) hermaphrodites were picked onto IPTG plates which had been seeded with the relevant bacterial RNAi feeding clones as previously described [56]. The oxa-1 feeding clone in the feeding RNAi bacterial strain HT115 was from the Ahringer Lab RNAi feeding library [57]. The $s f t-1$ feeding clone was made by inserting a PCR fragment corresponding to 400 bp of $s f t-1$ exonic sequence (amplified from fosmid H06104) into the L4440 RNAi feeding vector, using enzymes $\mathrm{XbaI}$ and HindIIII. This was electroporated into E. coli strain HT115 before feeding to worms.

\section{RT-PCR}

Reduction of the target transcripts following RNAi treatment was confirmed by gene-specific RT-PCR using the Superscript III RT system (Life Technologies (Invitrogen Division). Renfrew, Paisley, UK) with RNA from 20 L4 progeny from $s f t-1(R N A i)$ worms and six plates of progeny from oxa-1(RNAi) worms and equivalent N2 controls. Transcript levels of a non-target gene, eft-2, were measured as a control for the specificity of the RNAi. The RT reaction was primed using oligo $(\mathrm{dT})$ primers. RNA was extracted using a hot phenol protocol [58] and purified using a DNAse column. A total of $5 \mu \mathrm{L}$ of RNA dissolved in RNAse free water was used in the RT reaction to synthesize cDNA.
Specific sft-1, oxa-1and eft-2 RT-PCR primers were designed and amplified $220 \mathrm{bp}, 164 \mathrm{bp}$ and $350 \mathrm{bp}$ fragments, respectively. Twenty-five cycles of PCR were used in each case.

sft-1 F primer 5' GAAAGGGCGACTGAATCAAA 3' JH7

sft-1 R primer 5' GTGTCCTCCATGACTGCTCA 3' JH8

oxa-1 F primer 5' GCACTTCCATTCATCTCTGC 3' JH9

oxa-1 R primer 5' CATAGACCCGTAGCAAATTGTG 3' JH10

eft-2 F primer 5' GCGTATCAAGCCAGTTCTTT 3' JH19

eft-2 R primer 5' CTGCTCCACTTCTTGGTCTT 3' $\mathrm{JH} 20$

\section{Brood counts}

Brood sizes were assessed using 20 synchronized L4 worms each injected with dsRNA corresponding to $s f t-1$ or oxa-1. Control groups were injected with TE buffer only. (In the case of feeding RNAi experiments, L3 animals were first picked onto NGM plates seeded with HT115 bacteria transformed with the relevant feeding RNAi clone or empty vector control). For the brood size counts, L4 animals were picked singly onto individual 55 mm NGM plates seeded with OP50 bacteria (or HT115 transformed with the relevant feeding RNAi clone or empty vector control), and transferred to fresh seeded plates every 24 hours until egg laying had stopped. Counting of unhatched eggs and live progeny was performed on plates from which the mother had been moved the previous day and individual plate counts totaled in order to derive a complete brood size for each animal. All plates were kept at $20^{\circ} \mathrm{C}$.

\section{Cytochrome oxidase (COX) staining}

COX activity was assayed in cells of intact worms by the oxidation of diaminobenzidine in the presence of cytochrome c in a protocol adapted from Seligman et al. [59]. The specificity of the COX staining reaction was demonstrated by the absence of reaction product when worms were incubated with sodium azide $\left(\mathrm{NaN}_{3}\right)$. A total of 50 mixed-stage animals (either the progeny of animals injected with $s f t-1$ dsRNA, or controls) were picked into $50 \mu \mathrm{l}$ of M9 buffer in an Eppendorf tube (in duplicate) (Eppendorf UK Ltd, Stevenage, Herts, UK). The worms were allowed to settle and the M9 buffer was carefully removed. A total of $1 \mathrm{ml}$ of freshly prepared COX stain ( $1 \mathrm{mg} / \mathrm{mL}$ diaminobenzidine- $\mathrm{HCl}, 1 \mathrm{mg} / \mathrm{mL}$ cytochrome c, $2 \mathrm{mg} / \mathrm{mL}$ catalase in $50 \mathrm{mM}$ sodium phosphate buffer, $\mathrm{pH}$ 7.4) was added to the worms and gently mixed. Worms were incubated with the stain for $4 \mathrm{~h}$ at $20^{\circ} \mathrm{C}$. $\mathrm{NaN}_{3}$ was added to one of the duplicate tubes to a final concentration of $1 \mathrm{mM}$. The COX stain was removed and the worms gently rinsed with PBS twice. Tubes were spun at $6,000 \mathrm{rpm}$ for 8 secs to gently sediment the worms 
between rinses. The worms were then placed on a slide and allowed to air dry (approximately 30 minutes). They were subsequently mounted for microscopy by adding a coverslip coated with Aquatex (VWR International Ltd, Lutterworth, Leics, UK) and samples photographed using identical settings and exposure times. Experiments were repeated at least three times.

\section{Determination of lifespan}

Lifespan assays were performed using 20 to 50 worms for each strain (or F1 progeny of animals that had been injected with dsRNA corresponding to $s f t-1$ or oxa-1, or fed on the appropriate feeding RNAi bacteria) per experiment. Single L4 worms were picked onto individual wells of 12-well NGM plates seeded with OP50 bacteria, and transferred daily to a fresh seeded well until the end of egg laying. Animals were checked for viability every day by recording their response to mechanical stimulus. Worms that did not respond to five gentle prods with a platinum wire were scored as dead and the date of death recorded. All lifespan assays were performed at $20^{\circ} \mathrm{C}$, with three biological replicates.

\section{Sensitivity to oxidative stress}

Oxidative stress sensitivity assays were performed using 50 worms for each strain (or F1 progeny of animals that had been injected with dsRNA corresponding to $s f t-1$ or oxa-1).

$s f t-1$ (RNAi) or oxa-1(RNAi) worms and size-matched N2 controls were picked onto $30 \mathrm{~mm}$ NGM plates (seeded with OP50 bacteria, 10 animals per plate) containing final concentrations of 0,10 or $25 \mathrm{mM}$ paraquat (1,1'-Dimethyl-4,4'-bipyridinium dichloride hydrate, Sigma (Sigma-Aldrich, Poole, Dorset, UK)). Survival was assessed at regular intervals over the course of 100 hours by response to mechanical stimulus as described above. Animals that escaped from the plate and thus could not be accounted for were excluded from the analysis. All plates were kept at $20^{\circ} \mathrm{C}$.

\section{sft-1 and oxa-1 GFP reporter constructs}

$s f t-1$ and $o x a-1$ translational GFP fusion constructs were made using the PCR fusion method [60]. An 8,129 bp fragment containing the $s f t-1$ ORF (H06I04.2) and 3,204 bp of upstream sequence was amplified from fosmid H06104 using Expand Long Template polymerase (Roche Diagnostics Ltd., Burgess Hill, West Susses, UK) and primers JH13 and JH15. JH15 includes a tag complementary to sequence at the 5' end of GFP.

$s f t-1 \mathrm{~F}$ primer 5'GTAAGCTGGAATCGGCAAAG 3' JH13

sft-1 R primer 5' CGACCTGCAGGCATGCAAGCT CCACATGAGCATTGTGAC 3’ JH15.
A 6,870 bp fragment containing the oxa-1 ORF (C01A2.3) and 3,770 bp of upstream sequence (including all putative regulatory sequence elements within the operon CE0P1696) was amplified from fosmid I6F08, using Expand Long polymerase and primers JH16 and JH18. JH18 includes a tag complementary to sequence at the 5' end of GFP.

oxa-1 F primer 5' CGTCTCTCCCATCCAGCTT 3' $\mathrm{JH} 16$

oxa-1 R primer 5' CGACCTGCAGGCATGCAA GCATGAAGCATCACGTTGTCG 3' JH18

To fuse the gene specific PCR products to the GFP reporter, $0.5 \mu \mathrm{L}$ of each PCR product and $0.5 \mu \mathrm{L}$ of gel purified GFP PCR product ( $1.8 \mathrm{~kb}$ fragment, the product of PCR with primers ppdgfp and gfp $\mathrm{c} 1$ from plasmid pPD95.75 (Fire Lab vectors obtained from Addgene, Cambridge, MA, USA) were added to the Expand Long Buffer 3 system reaction mix to form the template for the sewing reaction. A second forward nested primer and GFP specific reverse primer (gfp c2) were used to amplify the full-length gene-GFP fused PCR product. These products were gel purified using the SYBR-RED gel purification system.

sft-1 nested forward primer: 5'GTGTATGCAAAT GCGACGAG 3' JH14

oxa-1 nested forward primer 5'CGTCTCTCCCATCC AGCTT 3' JH17

ppdgfp primer 5'GCTTGCATGCCTGCAGGTCG3' gfp c1 primer 5'AAGGGCCCGTACGGCCGACTAG TAGG3'

gfp c2 primer 5'AAACAGTTATGTTTGGTATATT GGG3'

The purified PCR products were cloned into the TOPO XL vector using the TOPO XL cloning kit (Invitrogen) and shown to contain the correct inserts by restriction digest and sequencing. The sft-1::gfp construct is pAW317 and the oxa-1::gfp construct is pAW318.

\section{Transgenic worms}

GFP reporter constructs were injected into the syncytial gonad of young adult hermaphrodite worms at a concentration of approximately $20 \mathrm{ng} / \mu \mathrm{L}$ as described [55] along with the rol-6 transformation marker $p$ Ces1943. Rol progeny were picked and stable lines selected for analysis. The $s f t-1:: g f p$ transgenic strain described in this study is AW240 (ouEx608 (pAW317 + rol-6)) and the oxa-1::gfp transgenic strain is AW241 (ouEx609 (pAW318 + rol-6)).

\section{Mitotracker staining}

L4 stage N2 and AW241 worms were transferred to seeded NGM plates with $2 \mu \mathrm{g} / \mathrm{ml}$ Mitotracker Red (Life Technologies Ltd (Invitrogen division, Renfrew, Paisley, UK) spread on the surface and stored in the dark. L4 
progeny from these animals were picked, washed in M9 for one hour and mounted for fluorescence microscopy as described above.

\section{Additional files}

\section{Additional file 1: Lifespan extension in sft-1(RNAi) and oxa-1(RNAi)} worms displays differential dependence on the insulin-like signalling pathway. A: Combined dataset for three separate experiments. Analytical values for lifespan experiments are shown, including mean and median adult lifespan, maximum adult lifespan and the sample size $(n)$ for each strain and experimental condition. Statistical tests (Log-Rank tests using OASIS software [61]) were carried out using the lifespan of each worm in the entire population. oxa-1(RNAi) and sft-1 (RNAi) animals have a significantly increased mean lifespan compared with the wild type $\mathrm{N} 2$ strain $\left(P=1 \times 10^{-7}\right.$ and $2 \times 10^{-5}$, respectively). daf16(m26); oxa-1(RNAi) animals have a significantly increased lifespan compared with daf-16 alone $\left(p<1 \times 10^{-10}\right)$, but the difference is not significant when compared with oxa-1 alone $(p=0.09)$. daf-16 $(\mathrm{m} 26)$; sft-1 (RNAi) animals do not have a significantly different lifespan from daf-16 alone ( $P=0.75)$ but these animals have a significantly decreased lifespan compared with sft-1(RNAi) alone $\left(P<1 \times 10^{-10}\right)$. B: Individual datasets for the three separate experiments. Mean, median and maximum lifespans are shown. Log-Rank tests were carried out using the lifespan of each worm in the entire population. ${ }^{*}$ denotes the significance $(P)$ value compared to N2 worms, ${ }^{* *}$ denotes the $P$-value compared to daf-16(m26) worms. oxa-1 (RNAi) animals had a significant lifespan extension compared to control animals in all three biological replicates which was at least partially independent of daf-16. sft-1(RNAi) animals had a significant lifespan extension compared to control animals in two out of three biological replicates (in the third replicate the $P$-value was 0.079). In all cases, the lifespan extension was dependent on daf-16 (that is, significant lifespan extension was not observed in a daf-16(m26) mutant background)

Additional file 2: Sensitivity of sft-1(RNAi) animals to oxidative stress. Analytical values for survival following exposure to different concentrations of paraquat are shown, including mean and median survival (in hours), standard error of the mean (SEM), maximum survival and the sample size (n) for each strain and experimental condition. Statistical tests ( $t$-tests) were carried out using the survival time of each worm in the population. sft-1(RNAi) animals do not have a significantly different mean survival time compared with N2 controls after exposure to either $10 \mathrm{mM}$ or $25 \mathrm{mM}$ paraquat ( $P=0.30$ and 0.41 , respectively).

\section{Additional file 3: Resistance of oxa-1(RNAi) animals to oxidative} stress. Analytical values for survival following exposure to different concentrations of paraquat are shown, including mean and median survival (in hours), standard error of the mean (SEM), maximum survival and the sample size (n) for each strain and experimental condition. Statistical tests ( $t$-tests) were carried out using the survival time of each worm in the population. oxa-1 (RNAi) animals displayed a significantly increased mean survival time compared with N2 controls following exposure to either $10 \mathrm{mM}$ or $25 \mathrm{mM}$ paraquat $\left(P=1 \times 10^{-25}\right.$ and $1 \times 10^{-7}$, respectively).

Additional file 4: sft-1::gfp expression pattern. A, B: Transgenic strain AW240 (ouEx608 (pAW317 + rol-6)), where pAW317 is a translational GFP fusion of sft-1 driven by the intercistronic promoter. sft- $1:: \mathrm{gfp}$ is expressed at a very low level throughout the worm, but is concentrated in particular tissues. Left hand images, DIC only, right hand images, DIC and GFP merged. A: sft-1::gfp expression in muscle surrounding the pharynx (white arrow). B: sft-1::gfp expression in the uterus (white arrowheads). Scale bar, $100 \mu \mathrm{m}$. Anterior is to the left in all images.

\section{Abbreviations}

COX: Cytochrome oxidase; MIT: Mitochondrial; NGM: Nematode growth media; PBS: Phosphate-buffered saline; ROS: Reactive oxygen species.

\section{Competing interests}

The authors declare that they have no competing interests.

\section{Authors' contributions}

$\mathrm{SM}, \mathrm{JH}, \mathrm{CB}$ and PA carried out the brood size, life span and oxidative stress assays. RB and CD performed the COX staining experiments, and SM, JH and RB constructed and analyzed the GFP expression patterns. GB and AW conceived of the study, participated in its design and coordination, and wrote the manuscript. All authors read and approved the final manuscript.

\section{Acknowledgements}

Some nematode strains used in this work were provided by the Caenorhabditis Genetics Center, which is funded by the NIH National Center for Research Resources (NCRR). This work was funded by grants to AW from the MRC (G0001282, and a Capacity Building Studentship) and to GB from the Wellcome Trust.

\section{Author details}

'Biochemistry Department, University of Oxford, South Parks Road, Oxford OX1 3QU, UK. 'Present address: CRUK London Research Institute, 44 Lincoln's Inn Fields, London WC2A 3LY, UK. ${ }^{3}$ Present address: Cytogenetics Department, South East Scotland Genetics Service, Western General Hospital, Edinburgh, UK.

Received: 11 June 2012 Accepted: 25 March 2013

Published: 08 May 2013

\section{References}

1. Gropman AL: The neurological presentations of childhood and adult mitochondrial disease: established syndromes and phenotypic variations. Mitochondrion 2004, 4:503-520.

2. Oswald C, Krause-Buchholz U, Rodel G: Knockdown of human COX17 affects assembly and supramolecular organization of cytochrome $c$ oxidase. J Mol Biol 2009, 389:470-479.

3. Zhu Z, Yao J, Johns T, Fu K, De Bie I, Macmillan C, Cuthbert AP, Newbold RF, Wang J, Chevrette M, Brown GK, Brown RM, Shoubridge EA: SURF1, encoding a factor involved in the biogenesis of cytochrome c oxidase, is mutated in Leigh syndrome. Nat Genet 1998, 20:337-343. Comment in: Nat Genet 1998, 20:316-317.

4. Bonnefoy N, Chalvet F, Hamel P, Slonimski PP, Dujardin G: OXA1, a Saccharomyces cerevisiae nuclear gene whose sequence is conserved from prokaryotes to eukaryotes controls cytochrome oxidase biogenesis. J Mol Biol 1994, 239:201-212

5. Ventura N, Rea SL, Testi R: Long-lived C. elegans mitochondrial mutants as a model for human mitochondrial-associated diseases. Exp Gerontol 2006, 41:974-991.

6. Rea SL, Graham BH, Nakamaru-Ogiso E, Kar A, Falk MJ: Bacteria, yeast, worms, and flies: exploiting simple model organisms to investigate human mitochondrial diseases. Dev Disabil Res Rev 2010, 16:200-218.

7. Ventura N, Rea SL: Caenorhabditis elegans mitochondrial mutants as an investigative tool to study human neurodegenerative diseases associated with mitochondrial dysfunction. Biotechnol J 2007, 2:584-595.

8. Van Raamsdonk JM, Hekimi S: Reactive oxygen species and aging in Caenorhabditis elegans: causal or casual relationship? Antioxid Redox Signal 2010, 13:1911-1953.

9. Rea SL, Ventura N, Johnson TE: Relationship between mitochondrial electron transport chain dysfunction, development, and life extension in Caenorhabditis elegans. PLoS Biol 2007, 5:e259.

10. Feng J, Bussiere F, Hekimi S: Mitochondrial electron transport is a key determinant of life span in Caenorhabditis elegans. Dev Cell 2001, 1:633-644.

11. Miyadera H, Amino H, Hiraishi A, Taka H, Murayama K, Miyoshi H, Sakamoto $\mathrm{K}$, Ishii N, Hekimi S, Kita K: Altered quinone biosynthesis in the long-lived clk-1 mutants of Caenorhabditis elegans. J Biol Chem 2001, 276:7713-7716.

12. Lee SS, Lee RY, Fraser AG, Kamath RS, Ahringer J, Ruvkun G: A systematic RNAi screen identifies a critical role for mitochondria in C. elegans longevity. Nat Genet 2003, 33:40-48.

13. Tsang WY, Sayles LC, Grad LI, Pilgrim DB, Lemire BD: Mitochondrial respiratory chain deficiency in Caenorhabditis elegans results in developmental arrest and increased life span. J Biol Chem 2001, 276:32240-32246.

14. Dillin A, Hsu AL, Arantes-Oliveira N, Lehrer-Graiwer J, Hsin H, Fraser AG, Kamath RS, Ahringer J, Kenyon C: Rates of behavior and aging specified by mitochondrial function during development. Science 2002, 298:2398-2401. 
15. Hamilton B, Dong Y, Shindo M, Liu W, Odell I, Ruvkun G, Lee SS: A systematic RNAi screen for longevity genes in C. elegans. Genes Dev 2005, 19:1544-1555.

16. Kayser EB, Sedensky MD, Morgan PG: The effects of complex i function and oxidative damage on lifespan and anesthetic sensitivity in Caenorhabditis elegans. Mech Ageing Dev 2004, 125:455-464.

17. Butler JA, Ventura N, Johnson TE, Rea SL: Long-lived mitochondrial (Mit) mutants of Caenorhabditis elegans utilize a novel metabolism. FASEB J 2010, 24:4977-4988.

18. Van Raamsdonk JM, Meng Y, Camp D, Yang W, Jia X, Benard C, Hekimi S: Decreased energy metabolism extends life span in Caenorhabditis elegans without reducing oxidative damage. Genetics 2010, 185:559-571.

19. Lee $S$ J, Hwang $A B$, Kenyon $C$ : Inhibition of respiration extends $C$. elegans life span via reactive oxygen species that increase HIF-1 activity. Curr Biol 2010, 20:2131-2136

20. Yang W, Hekimi S: A mitochondrial superoxide signal triggers increased longevity in Caenorhabditis elegans. PLOS Biol 2010, 8:e1000556.

21. Heidler T, Hartwig K, Daniel H, Wenzel U: Caenorhabditis elegans lifespan extension caused by treatment with an orally active ROS-generator is dependent on DAF-16 and SIR-2.1. Biogerontology 2010, 11:183-195.

22. Van Raamsdonk JM, Hekimi S: Deletion of the mitochondrial superoxide dismutase sod-2 extends lifespan in Caenorhabditis elegans. PLoS Genet 2009, 5:e1000361.

23. Hwang $A B$, Lee $S J$ : Regulation of life span by mitochondrial respiration: the HIF-1 and ROS connection. Aging (Albany NY) 2011, 3:304-310.

24. Ristow M, Zarse K: How increased oxidative stress promotes longevity and metabolic health: the concept of mitochondrial hormesis (mitohormesis). Exp Gerontol 2010, 45:410-418.

25. Tiranti V, Hoertnagel K, Carrozzo R, Galimberti C, Munaro M, Granatiero M, Zelante L, Gasparini P, Marzella R, Rocchi M, Bayona-Bafaluy MP, Enriquez JA Uziel G, Bertini E, Dionisi-Vici C, Franco B, Meitinger T, Zeviani M: Mutations of SURF-1 in Leigh disease associated with cytochrome c oxidase deficiency. Am J Hum Genet 1998, 63:1609-1621.

26. Tiranti V, Galimberti C, Nijtmans L, Bovolenta S, Perini MP, Zeviani M: Characterization of SURF-1 expression and Surf-1p function in normal and disease conditions. Hum Mol Genet 1999, 8:2533-2540

27. Jia L, Dienhart M, Schramp M, McCauley M, Hell K, Stuart RA: Yeast Oxa1 interacts with mitochondrial ribosomes: the importance of the C-terminal region of Oxa1. EMBO J 2003, 22:6438-6447.

28. Kenyon C, Chang J, Gensch E, Rudner A, Tabtiang R: A C. elegans mutant that lives twice as long as wild type. Nature 1993, 366:461-464.

29. Dorman JB, Albinder B, Shroyer T, Kenyon C: The age- 1 and daf-2 genes function in a common pathway to control the lifespan of Caenorhabditis elegans. Genetics 1995, 141:1399-1406.

30. Ishii N, Takahashi K, Tomita S, Keino T, Honda S, Yoshino K, Suzuki K: A methyl viologen-sensitive mutant of the nematode Caenorhabditis elegans. Mutat Res 1990, 237:165-171.

31. Allen MA, Hillier LW, Waterston $R H$, Blumenthal T: A global analysis of $C$. elegans trans-splicing. Genome Res 2011, 21:255-264.

32. Bonnefoy N, Kermorgant M, Groudinsky O, Dujardin G: The respiratory gene OXA1 has two fission yeast orthologues which together encode a function essential for cellular viability. Mol Microbiol 2000, 35:1135-1145.

33. Bonnefoy N, Kermorgant M, Groudinsky O, Minet M, Slonimski PP, Dujardin $\mathrm{G}$ : Cloning of a human gene involved in cytochrome oxidase assembly by functional complementation of an oxa1- mutation in Saccharomyces cerevisiae. Proc Natl Acad Sci U S A 1994, 91:11978-11982.

34. Altamura N, Capitanio N, Bonnefoy N, Papa S, Dujardin G: The Saccharomyces cerevisiae OXA1 gene is required for the correct assembly of cytochrome c oxidase and oligomycin-sensitive ATP synthase. FEBS Lett 1996, 382:111-115.

35. Bonnefoy N, Fiumera HL, Dujardin G, Fox TD: Roles of Oxa1-related innermembrane translocases in assembly of respiratory chain complexes. Biochim Biophys Acta 2009, 1793:60-70.

36. Vanfleteren JR, De Vreese A: Rate of aerobic metabolism and superoxide production rate potential in the nematode Caenorhabditis elegans. J Exp Zool 1996, 274:93-100.

37. Braeckman BP, Houthoofd K, Vanfleteren JR: Intermediary metabolism. WormBook 2009, 16:1-24.

38. Dernburg AF, Zalevsky J, Colaiacovo MP, Villeneuve AM: Transgene-mediated cosuppression in the $C$. elegans germ line. Genes Dev 2000, 14:1578-1583.

39. Lakowski B, Hekimi S: The genetics of caloric restriction in Caenorhabditis elegans. Proc Natl Acad Sci U S A 1998, 95:13091-13096.
40. Felkai S, Ewbank JJ, Lemieux J, Labbe JC, Brown GG, Hekimi S: CLK-1 controls respiration, behavior and aging in the nematode Caenorhabditis elegans. EMBO J 1999, 18:1783-1792.

41. Mukhopadhyay A, Oh SW, Tissenbaum HA: Worming pathways to and from DAF-16/FOXO. Exp Gerontol 2006, 41:928-934.

42. Ishii N, Fujii M, Hartman PS, Tsuda M, Yasuda K, Senoo-Matsuda N, Yanase S, Ayusawa D, Suzuki K: A mutation in succinate dehydrogenase cytochrome b causes oxidative stress and ageing in nematodes. Nature 1998, 394:694-697.

43. Tsang WY, Lemire BD: The role of mitochondria in the life of the nematode, Caenorhabditis elegans. Biochim Biophys Acta 2003, 1638:91-105.

44. Ristow M, Schmeisser S: Extending life span by increasing oxidative stress. Free Radic Biol Med 2011, 51:327-336.

45. Schulz TJ, Zarse K, Voigt A, Urban N, Birringer M, Ristow M: Glucose restriction extends Caenorhabditis elegans life span by inducing mitochondrial respiration and increasing oxidative stress. Cell Metab 2007, 6:280-293.

46. Yang W, Hekimi S: Two modes of mitochondrial dysfunction lead independently to lifespan extension in Caenorhabditis elegans. Aging Cell 2010, 9:433-447

47. Zuryn S, Kuang J, Tuck A, Ebert PR: Mitochondrial dysfunction in Caenorhabditis elegans causes metabolic restructuring, but this is not linked to longevity. Mech Ageing Dev 2010, 131:554-561.

48. Gallo M, Park D, Riddle DL: Increased longevity of some C. elegans mitochondrial mutants explained by activation of an alternative energy-producing pathway. Mech Ageing Dev 2011, 132:515-518.

49. Cristina D, Cary M, Lunceford A, Clarke C, Kenyon C: A regulated response to impaired respiration slows behavioral rates and increases lifespan in Caenorhabditis elegans. PLoS Genet 2009 5:e1000450.

50. Lapointe J, Hekimi S: Early mitochondrial dysfunction in long-lived Mclk1 +/- mice. J Biol Chem 2008, 283:26217-26227.

51. Dell'agnello C, Leo S, Agostino A, Szabadkai G, Tiveron C, Zulian A, Prelle A Roubertoux P, Rizzuto R, Zeviani M: Increased longevity and refractoriness to $\mathrm{Ca}(2+)$-dependent neurodegeneration in Surf1 knockout mice. Hum Mol Genet 2007, 16:431-444.

52. Zordan MA, Cisotto P, Benna C, Agostino A, Rizzo G, Piccin A, Pegoraro M, Sandrelli F, Perini G, Tognon G, De Caro R, Peron S, Kronniè $\Pi$, Megighian A, Reggiani C, Zeviani M, Costa R: Posttranscriptional silencing and functional characterization of the Drosophila melanogaster homolog of human Surf1. Genetics 2006, 172:229-241

53. Sulston J, Hodgkin J: Methods. In The Nematode Caenorhabditis elegans. Ith edition. Edited by Wood WB. New York: Cold Spring Harbor Laboratory Press; 1988:587-606.

54. Fire A, Xu S, Montgomery MK, Kostas SA, Driver SE, Mello CC: Potent and specific genetic interference by double-stranded RNA in Caenorhabditis elegans. Nature 1998, 391:806-811.

55. Mello C, Fire A: DNA transformation. Methods Cell Biol 1995, 48:451-482.

56. Kamath RS, Ahringer J: Genome-wide RNAi screening in Caenorhabditis elegans. Methods 2003, 30:313-321.

57. Kamath RS, Fraser AG, Dong $Y$, Poulin G, Durbin R, Gotta M, Kanapin A, Le Bot N, Moreno S, Sohrmann M, Welchman DP, Zipperlen P, Ahringer J: Systematic functional analysis of the Caenorhabditis elegans genome using RNAi. Nature 2003, 421:231-237.

58. Furger A, Monks J, Proudfoot NJ: The retroviruses human immunodeficiency virus type 1 and Moloney murine leukemia virus adopt radically different strategies to regulate promoter-proxima polyadenylation. J Virol 2001, 75:11735-11746.

59. Seligman AM, Karnovsky MJ, Wasserkrug HL, Hanker JS: Nondroplet ultrastructural demonstration of cytochrome oxidase activity with a polymerizing osmiophilic reagent, diaminobenzidine (DAB). J Cell Biol 1968, 38:1-14

60. Hobert O: PCR fusion-based approach to create reporter gene constructs for expression analysis in transgenic C. elegans. Biotechniques 2002, 32:728-730.

61. Yang JS, Nam HJ, Seo M, Han SK, Choi Y, Nam HG, Lee SJ, Kim S: OASIS: online application for the survival analysis of lifespan assays performed in aging research. PLoS One 2011, 6:e23525

doi:10.1186/2046-2395-2-9

Cite this article as: Maxwell et al: The SFT-1 and OXA-1 respiratory chain complex assembly factors influence lifespan by distinct mechanisms in C. elegans. Longevity \& Healthspan 2013 2:9. 